\title{
Glucocorticoid Receptor and FKBP5 Expression Is Altered Following Exposure to Chronic Stress: Modulation by Antidepressant Treatment
}

\author{
Gianluigi Guidotti ${ }^{1,4}$, Francesca Calabrese ${ }^{1,4}$, Christoph Anacker ${ }^{2}$, Giorgio Racagni' ${ }^{1,3}$, Carmine M Pariante ${ }^{2}$ \\ and Marco A Riva*,1,3 \\ 'Dipartimento di Scienze Farmacologiche e Biomolecolari, Centro di Neurofarmacologia, Università degli Studi di Milano, Milan, Italy; ${ }^{2}$ Section of \\ Perinatal Psychiatry and Stress, Psychiatry and Immunology (SPI-lab), Department of Psychological Medicine, King's College London, Institute of \\ Psychiatry, London, UK; ${ }^{3}$ Center of Excellence on Neurodegenerative Diseases, Università degli Studi di Milano, Milan, Italy
}

Major depression is thought to originate from the interaction between susceptibility genes and adverse environmental events, in particular stress. The hypothalamus-pituitary-adrenal (HPA) axis is the major system involved in stress response and its dysregulation is an important element in the pathogenesis of depression. The stress response is therefore finely tuned through a series of mechanisms that control the trafficking of glucocorticoid receptors (GRs) to the nucleus, including binding to the chaperone protein FKBP5 and receptor phosphorylation, suggesting that these elements may also be affected under pathologic conditions. On these bases, we investigated FKBP5 and GR expression and phosphorylation in the hippocampus (ventral and dorsal) and in the prefrontal cortex of rats exposed to chronic mild stress (CMS) and we analyzed the effect of a concomitant antidepressant treatment. We found that animals exposed to CMS show increased expression of FKBP5 as well as enhanced cytoplasmic levels of GR, primarily in ventral hippocampus and prefrontal cortex. Chronic treatment with the antidepressant duloxetine is able to normalize such alterations, mainly in the prefrontal cortex. Moreover, we demonstrate that CMS-induced alterations of GR trafficking and transcription may be sustained by changes in receptor phosphorylation, which are also modulated by pharmacological intervention. In summary, while GR-related changes after CMS might be relevant for the depressive phenotype, the ability of antidepressant treatment to correct some of these alterations may contribute to the normalization of HPA axis dysfunctions associated with stress-related disorders.

Neuropsychopharmacology (20I3) 38, 6I6-627; doi:I0.1038/npp.20 I2.225; published online 2I November 2012

Keywords: depression; HPA axis; chronic mild stress; duloxetine

\section{INTRODUCTION}

Exposure to stress represents a major environmental contributor to the development of psychiatric conditions, such as major depression (Pittenger and Duman, 2008). Under physiological conditions, stress exposure activates the hypothalamus-pituitary-adrenal (HPA) axis, which leads to glucocorticoid (GC) release from the adrenal glands. The activation of the HPA axis is controlled through a negative feedback mechanism, by the activation of glucocorticoid receptors (GRs) at different levels, including the hippocampus (McEwen et al, 1992). However, under pathological conditions, the function of the HPA axis is

*Correspondence: Professor MA Riva, Department of Pharmacological and Biomolecular Sciences, Center of Neuropharmacology, University of Milan, Via Balzaretti 9, 20133 Milan, Italy, Tel: +39 0250318334 , Fax: + 3902 50318278, E-mail:m.riva@unimi.it

${ }^{4}$ These authors contributed equally to this work.

Received 28 June 2012; revised 17 September 2012; accepted 10 October 2012 disrupted, and this may eventually lead to structural and functional changes in brain regions that have a major role in the pathophysiology of depression (de Kloet et al, 2005; Holsboer, 2000).

While the cause of altered HPA axis function in depression is not known, several evidence indicate that negative feedback control of corticotrophin-releasing hormone (CRH) secretion may be impaired because of altered GR function in the hypothalamus and in the hippocampus (Pariante, 2006). This inability of GCs to exert their effects on these targets leads to the so-called 'glucocorticoid resistance', characterized by increased $\mathrm{CRH}$ production and over-activity of the HPA axis. Indeed, preclinical studies have demonstrated that GR heterozygous mutant mice $\left(\mathrm{GR}^{+/-}\right)$have a predisposition for depression (Ridder et al, 2005), supporting the hypothesis that reduced expression and function of GRs may be relevant for the pathogenesis of stress-related psychiatric disorders (Holsboer, 2000; de Kloet et al, 2005). Such changes are supported by clinical evidence, since both a reduction in the number and/or function of GR and abnormal patterns of 
cortisol secretion leading to increased $24 \mathrm{~h}$ production of cortisol, have been described in people suffering from major depression (Pariante and Lightman, 2008).

GRs act as a ligand-activated transcription factor that, upon hormone binding, translocate from the cytosol to the nucleus. This process, and the subsequent control on gene transcription, is regulated by a large chaperone protein complex consisting of FK506 binding protein 51 (FKBP5), heat-shock protein 70 (HSP70), and heat-shock protein 90 (HSP90). When FKBP5 is bound to the GR complex via HSP90, the receptor has lower affinity for its ligand and is retained in the cytoplasm; upon GC binding, GR dissociates from the chaperone complex, dimerizes, and translocates into the nucleus (Binder, 2009). GR translocation and transcriptional activity is also regulated by a complex pattern of phosphorylation on different serine residues of the receptor (Galliher-Beckley et al, 2008; Takabe et al, 2008; Wang et al, 2002). Importantly, recent work from some of the authors of the present paper (Anacker et al, 2011) has recently demonstrated that GC hormones and antidepressants indeed regulate GR function via different phosphorylation of the GR, subsequently leading to phosphorylation-dependent changes in GR-mediated gene transcription. Based on the role exerted by GR and the HPA axis in depression, the investigation of the mechanisms controlling GR activation represents an important issue to understand the molecular underpinnings contributing to HPA axis dysfunction in stress-related disorders and to establish the potential of pharmacological intervention in normalizing such alterations.

Based on these premises, the aim of our study was to characterize the alteration of GR and FKBP5, at the transcriptional, translational and post-translational level, in the chronic mild stress (CMS) model of depression (Calabrese et al, 2012), and to establish the impact of chronic antidepressant treatment on the observed changes, focusing on hippocampus and prefrontal cortex, two key regions for major depression and stress-related disorders.

\section{MATERIALS AND METHODS}

Reagents were purchased from Sigma-Aldrich (Milan, Italy), Bio-Rad Laboratories S.r.l. Italia (Segrate, Italy), Roche (Monza, Italy), Eurofins MWG-Operon, (Ebersberg, Germany), Tebu-bio (Magenta, Italy), Life technologies (Monza, Italy), GE Healthcare Europe GmbH (Pero, Italy), Abcam (Prodotti Gianni, Milan, Italy), and DAKO (Glostrup, Denmark).

\section{Animals}

Adult (3 months old) male Sprague-Dawley rats (Charles River, Calco, Italy) weighing 225-250 g were used throughout the experiments. Rats were housed in groups of three per cage under standard conditions ( $12 \mathrm{~h}$ light/dark cycle, light off at $1900 \mathrm{~h}$ ) and were exposed to daily handling for 2 weeks before any procedure. All animal handling and experimental procedures were performed in accordance with the EC (EEC Council Directive 86/609 1987), the Italian legislation on animal experimentation (Decreto Legislativo 116/92), and the National Institutes of Health Guide for the Care and Use of

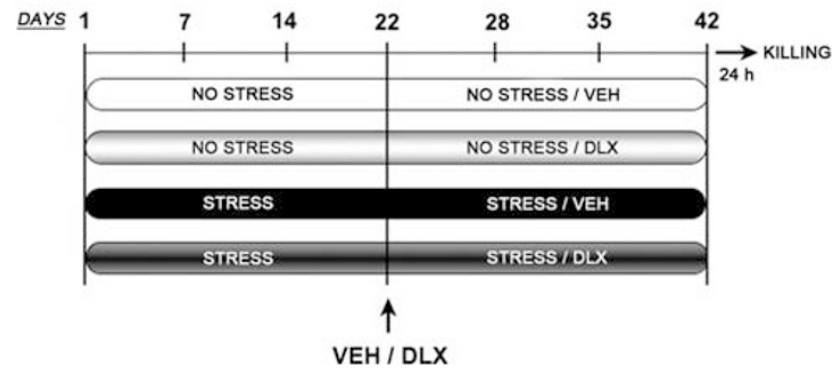

Figure I Schematic representation of the experimental paradigm used in the study. NO STRESS, sham animals; STRESS, animals exposed to chronic mild stress (CMS) paradigm. VEH, animals treated with vehicle (I\% HEC, I ml/ $/ \mathrm{kg})$; DLX, animals treated with duloxetine $(10 \mathrm{mg} / \mathrm{kg}$ daily). See Materials and methods for complete description.

Laboratory Animals. All efforts were made to minimize animal suffering and to reduce the number of animals used.

\section{Chronic Stress Paradigm and Antidepressant Treatment}

The experimental design is presented in Figure 1. Control animals (No Stress) were housed under standard conditions, whereas stressed animals were exposed to the chronic stress procedure (CMS) for a period of 42 consecutive days, a manipulation that leads to a chronic depressive-like state that develops gradually over time (Calabrese et al, 2012). The CMS regimen consisted of once or twice daily exposure to different stressors including food or water deprivation, crowding, isolation, soiled caged, $2 \mathrm{~h}$ immobilization, light on overnight. After 21 days, both stressed and control animals were each further divided into matched subgroups (n. 10-12 rats per group), and for subsequent 21 days they received daily oral administration (by gavage) of the antidepressant duloxetine, a serotonin and noradrenaline reuptake inhibitor (SNRI) $(10 \mathrm{mg} / \mathrm{kg}$ daily) or vehicle (hydroxyethylcellulose, HEC, $1 \%, 1 \mathrm{ml} / \mathrm{kg}$ ). Duloxetine is an SNRI widely used as antidepressant in human therapy (Frampton and Plosker, 2007). The dose selected in the present study is based on our previous work demonstrating the ability of chronic duloxetine treatment to effectively modulating neuronal plasticity (Calabrese et al, 2007; Molteni et al, 2009) as well as its normalizing properties in animals with genetic deletion of the serotonin transporter (Calabrese et al, 2010; Guidotti et al, 2012). On day 43, in the morning, rats were killed by decapitation $24 \mathrm{~h}$ after the last duloxetine administration. And brain regions were rapidly dissected, frozen on dry ice and stored at $-80^{\circ} \mathrm{C}$ for further analysis. The prefrontal cortex (defined as $\mathrm{Cg} 1, \mathrm{Cg} 3$, and IL subregions corresponding to the plates 6-10 according to the atlas of Paxinos and Watson, 1996) was dissected from $2 \mathrm{~mm}$-thick slices, whereas the hippocampus was dissected from $2 \mathrm{~mm}$ thick slices corresponding to plates 25-33 (dorsal hippocampus) and plates $34-43$ (ventral hippocampus) according to the atlas of Paxinos and Watson.

\section{Sucrose Preference Test}

The sucrose preference test was performed on the 21st day. For this aim, animals were initially exposed for $48 \mathrm{~h}$ to a palatable sucrose solution (1\%) to avoid neophobia. On day 
$21,12 \mathrm{~h}$ after water deprivation, sucrose preference was then determined by $1 \mathrm{~h}$ exposure to two identical bottles filled with either sucrose solution or water. Sucrose preference was defined as the ratio of the volume of sucrose $v s$ total liquid (water + sucrose) consumed during the 1-h test. We did not perform the test at the end of the treatment, ie, before the killing, because of the possible influence exerted by sucrose consumption on the expression of the molecular targets under investigation.

\section{RNA Preparation and Gene Expression Analysis by Quantitative Real-Time PCR}

Total RNA was isolated by single step of guanidinium isothiocyanate/phenol extraction using PureZol RNA isolation reagent (Bio-Rad Laboratories) according to manufacturer's instructions and quantified by spectrophotometric analysis. Following total RNA extraction, the samples were processed for real-time PCR (RT-PCR) to assess FKBP5 and Nr3c1 mRNA levels. An aliquot of each sample was treated with DNase to avoid DNA contamination. RNA was analyzed by TaqMan qRTPCR instrument (CFX384 real time system; Bio-Rad Laboratories) using the iScriptTM one-step RT-PCR kit for probes (Bio-Rad Laboratories). Samples were run in 384 well formats in triplicate as multiplexed reactions with a normalizing internal control (36B4). Primers and probes sequences used (Table 1) were purchased from Eurofins MWG-Operon and Life Technologies.

Thermal cycling was initiated with an incubation at $50{ }^{\circ} \mathrm{C}$ for $10 \mathrm{~min}$ (RNA retrotranscription) and then at $95^{\circ} \mathrm{C}$ for 5 min (TaqMan polymerase activation). After this initial step, 39 cycles of PCR were performed. Each PCR cycle consisted of heating the samples at $95^{\circ} \mathrm{C}$ for $10 \mathrm{~s}$ to enable the melting process and then for $30 \mathrm{~s}$ at $60^{\circ} \mathrm{C}$ for the annealing and extension reactions. A comparative cycle threshold (Ct) method was used to calculate the relative target gene expression.

\section{Analysis of FKBP5 and GR Protein Levels and GR Phosphorylation}

Western blot analysis was used to investigate FKBP5, GR, and three GR phospho-proteins in the nuclear and in the cytosolic fraction. Tissues were manually homogenized using a glass-glass potter in a $\mathrm{pH} 7.4$ cold buffer containing $0.32 \mathrm{~m}$ sucrose, $0.1 \mathrm{~mm}$ EGTA, $1 \mathrm{mM}$ HEPES solution in presence of a complete set of protease (Roche) and phosphatase (Sigma-Aldrich) inhibitors. The total homogenate was centrifuged at 2500 r.p.m. for $10 \mathrm{~min}$ at $4{ }^{\circ} \mathrm{C}$ to obtain the pellet corresponding to the nuclear fraction which was resuspended in a buffer (20 mM HEPES, $0.1 \mathrm{~mm}$ dithiothreitol (DTT), $0.1 \mathrm{~mm}$ EGTA) with protease and phosphatase inhibitors. The supernatant obtained was centrifuged at $10000 \mathrm{~g}$ for $15 \mathrm{~min}$ at $4{ }^{\circ} \mathrm{C}$ and the supernatant obtained correspond to the cytosolic fraction. The purity of subcellular fractions was assayed by antiglyceraldehyde 3-phosphate dehydrogenase (GAPDH) or anti-histone $\mathrm{H} 3$ antibodies for the cytoplasmic or nuclear compartments respectively, as shown in Figure 6d. Total protein content was measured according to the Bradford Protein Assay procedure (Bio-Rad Laboratories), using bovine serum albumin as calibration standard. Equal amounts of protein were run under reducing conditions on $10 \%$ SDS-polyacrylamide gels and then electrophoretically transferred onto nitrocellulose membranes (Bio-Rad Laboratories). The blots were blocked with $10 \%$ non-fat dry milk and then incubated with the primary antibodies summarized in Table 2. Membranes were then incubated

Table 2 Antibodies Conditions used in the Western Blot Analysis.

\begin{tabular}{|c|c|c|}
\hline Gene & Primary antibody & Secondary antibody \\
\hline FKBP5 & $\begin{array}{c}\text { I:2000 (Abcam) } \\
4^{\circ} \mathrm{C}, \mathrm{O} / \mathrm{N}\end{array}$ & $\begin{array}{l}\text { Anti-rabbit, } \\
\text { I:2500, RT, I h }\end{array}$ \\
\hline GR & $\begin{array}{c}\text { I: } 500 \text { (Thermo Scientific), } \\
4^{\circ} \mathrm{C}, \mathrm{O} / \mathrm{N}\end{array}$ & $\begin{array}{l}\text { Anti-rabbit, } \\
\text { I:2000, RT, I h }\end{array}$ \\
\hline Phospho GR S224 & $\begin{array}{l}1: 10000 \\
4^{\circ} \mathrm{C}, \mathrm{O} / \mathrm{N}\end{array}$ & $\begin{array}{l}\text { Anti-rabbit, } \\
\text { I: } 2000, \text { RT, I h }\end{array}$ \\
\hline Phospho GR S232 & $\begin{array}{c}\text { I: } 500(\text { Abcam }) \\
4^{\circ} \mathrm{C}, \mathrm{O} / \mathrm{N}\end{array}$ & $\begin{array}{l}\text { Anti-rabbit, } \\
\text { I: } 2000, \text { RT, I h }\end{array}$ \\
\hline Phospho GR S246 & $\begin{array}{c}1: 2000 \\
4^{\circ} \mathrm{C}, \mathrm{O} / \mathrm{N}\end{array}$ & $\begin{array}{l}\text { Anti-rabbit, } \\
\text { I: 2000, RT, I h }\end{array}$ \\
\hline$\beta$-Actin & $\begin{array}{l}\text { I : I } 10000 \text { (Sigma), } \\
4^{\circ} \mathrm{C}, \mathrm{O} / \mathrm{N}\end{array}$ & $\begin{array}{c}\text { Anti-mouse, } \\
\text { I: I0000, RT, I h }\end{array}$ \\
\hline
\end{tabular}

Table I Sequences of Forward and Reverse Primers and Probes used in Real-time PCR Analyses and Purchased from Eurofins MWG-Operon (a) and from Life Technologies, Which did not Disclose the Sequences (b).

(a) Forward and reverse primers and probes purchased from Eurofins MWG-Operon

\begin{tabular}{|c|c|c|c|}
\hline Gene & Forward primer & Reverse primer & Probe \\
\hline Fkbp5 & GAACCCAATGCTGAGCTTATG & ATGTACTTGCCTCCCTTGAAG & TGTCCATCTCCCAGGATTCTITGGC \\
\hline $\mathrm{Nr} 3 \mathrm{cl}$ & GAAAAGCCATCGTCAAAAGGG & TGGAAGCAGTAGGTAAGGAGA & AGCTाTGTCAGTTGGTAAAACCGTTGC \\
\hline Foxol & GAGTGGATGGTGAAGAGTGTG & GGACAGATTGTGGCGAATTG & TCAAGGATAAGGGCGACAGCAACAG \\
\hline$p||$ & AGAGTGCTCATGGAAAGGGA & AGCTCTGGAAGCCCACTाTT & ATAATGAAAGACCTGGACCAGTGC \\
\hline 36B4 & TTCCCACTGGCTGAAAAGGT & CGCAGCCGCAAATGC & AAGGCCTTCCTGGCCGATCCATC \\
\hline \multicolumn{4}{|c|}{ (b) Forward and reverse primers and probes purchased from Life Technologies } \\
\hline Gene & Accession number & Assay ID & \\
\hline Gadd $45 \beta$ & BC085337.I & Rn0|452530_gl & \\
\hline
\end{tabular}


for $1 \mathrm{~h}$ at room temperature with the opportune secondary antibody (see Table 2), and immunocomplexes were visualized by chemiluminescence using the ECL Western Blotting kit (GE Healthcare Europe $\mathrm{GmbH}$ ).

For GR phosphorylation analysis, protein samples containing $30 \mu \mathrm{g}$ of total protein were boiled for $10 \mathrm{~min}$ at $72{ }^{\circ} \mathrm{C}$ in $1 \times$ NuPAGE LDS sample buffer (Life Technologies) and $1 \times$ NuPAGE sample reducing agent (Life Technologies), and subjected to reducing SDS-PAGE on 10\% NuPAGE BisTris gels for $1 \mathrm{~h}$ at $200 \mathrm{~V}$. Proteins were electrophoretically transferred onto Immuno-Blot PVDF membranes (Bio-Rad Laboratories) at $110 \mathrm{~V}$ for $1.5 \mathrm{~h}$ at $4{ }^{\circ} \mathrm{C}$. Transfer efficiency was controlled by Ponceau $\mathrm{S}$ staining and by prestained protein standards. Unspecific binding sites were blocked for $1 \mathrm{~h}$ in $5 \%$ BSA in TBS and membranes were immunoprobed with the polyclonal rabbit anti-P-S224 $(1: 10000)$ and anti-P-S246 $(1: 2000)$ antibodies (both from Dr Michael J Garabedian), anti-P-S232 antibody (1:500; Abcam) in blocking solution at $4{ }^{\circ} \mathrm{C}$ overnight. Membranes were washed with TBS containing $0.1 \%$ Tween-20 (TBST) and incubated with an HRP-conjugated swine anti-rabbit secondary antibody $(1: 2000 ; \mathrm{DAKO})$, in $5 \%$ non-fat dry milk in TBS, for $1 \mathrm{~h}$ at room temperature. Membranes were washed in TBST and proteins were visualized with enhanced chemiluminescence (ECL) detection system (GE Healthcare, UK).

Results were standardized using $\beta$-actin as the control protein, which was detected by evaluating the band density at $43 \mathrm{kDa}$. Protein levels were calculated by measuring the optical density of the autoradiographic bands using Quantity One software (Bio-Rad Laboratories). To ensure that autoradiographic bands were in the linear range of intensity, different exposure times were used.

\section{Statistical Analyses}

The effects of stress and/or antidepressant treatment were analyzed with student's $t$-test, with a two-way analysis of variance (ANOVA) followed by Single Contrast Post Hoc Test (SCPHT). Significance for all tests was assumed for $P<0.05$. Data are presented as mean values \pm standard error (SEM). For graphic clarity, results are presented as mean percent of No Stress/Vehicle-treated rats.

\section{RESULTS}

\section{Effects of CMS on Body Weight and Behavior}

Before starting the antidepressant treatment, we established the effectiveness of the adverse manipulation by measuring body weight and anhedonia. As shown in Figure 2a, animals exposed to 3 weeks of CMS showed significantly less weight gain when compared with control animals $(P<0.001)$, an effect that may be due to reduced food and water consumption (data not shown). Moreover, as shown in Figure $2 \mathrm{~b}$, we found that rats exposed for 3 weeks to the CMS display a significantly reduced preference for sucrose solution $(-16 \%, P<0.05)$, when compared with nonstressed rats. Overall, these changes are clear indicators for the efficacy of the stressful manipulation.
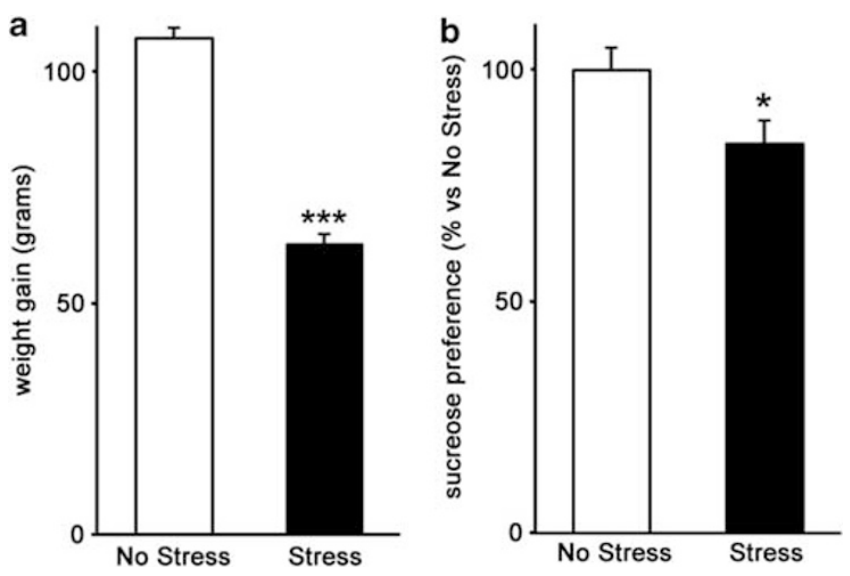

Figure 2 Effects of chronic mild stress (CMS) on rat weight gain and sucrose preference. (a) Animals showed a significant decrease in body weight gain after 3 weeks of CMS, when compared with no stress animals. The data, expressed as weight difference between day 21 and day I, represent the mean \pm SEM of at least 33 independent determinations. **** $P<0.001$ vs No Stress (Student's t-test). (b) Analysis of sucrose preference. Animals were initially exposed for $48 \mathrm{~h}$ to a palatable sucrose solution (I\%) to avoid neophobia. On day 2I, I2 h after water deprivation, sucrose preference was then determined by I h exposure to two identical bottles filled with either sucrose solution or water. Sucrose preference was defined as the ratio of the volume of sucrose vs total liquid (water + sucrose) consumed during the I-h test. $* P<0.05$ vs No Stress (Student's t-test).

\section{Modulation of FKBP5 mRNA and Protein Expression}

We initially investigated whether exposure to CMS could alter mRNA and protein levels of the hsp90 co-chaperone FKBP5, and to what extent chronic antidepressant treatment may affect the changes produced by stress exposure.

The modulation of FKBP5 was strictly area dependent. In fact, as shown in Figure 3a, Fkbp5 mRNA levels in the ventral hippocampus were significantly regulated by stress $\left(\mathrm{F}_{1,48}=24.90, P<0.001\right)$ with a significant stress $\times$ treatment interaction $\left(\mathrm{F}_{1,48}=16.40, P<0.001\right)$. Indeed, Fkbp5 expression was significantly increased following CMS $(+213 \%, P<0.001$ vs No Stress/Veh animals). Duloxetine produced a significant increase in Fkbp 5 mRNA levels when given to control rats $(+102 \%, P<0.05 v s$ No Stress/Veh animals), but reduced it when administered to CMS rats $(-29 \%, P<0.05$ vs Stress/Veh animals). In the dorsal hippocampus (Figure $3 \mathrm{~b}$ ), we found a significant effect of chronic stress $\left(\mathrm{F}_{1,47}=14.11, \quad P<0.001\right)$. Again, Fkbp5 expression was significantly increased after CMS exposure ( $+69 \%, P<0.01$, vs No Stress/Veh animals), an effect that was not influenced by duloxetine administration. Then, we analyzed the modulation of Fkbp5 in the prefrontal cortex (Figure 3c) and again we found a significant stress $\times$ treatment interaction $\left(\mathrm{F}_{1,47}=9.55, P<0.01\right)$. In fact, similarly to ventral and dorsal hippocampus, CMS produced a significant increase in Fkbp5 mRNA levels $(+102 \%$, $P<0.01$, vs No Stress/Veh animals). Chronic duloxetine reduced Fkbp5 mRNA levels in CMS rats $(-44 \%, P<0.05$, $v s$ Stress/Veh animals), while increasing its expression in control animals, although this change did not reach statistical significance due to some variability within the group of sham rats treated with duloxetine $(+42 \%$, 

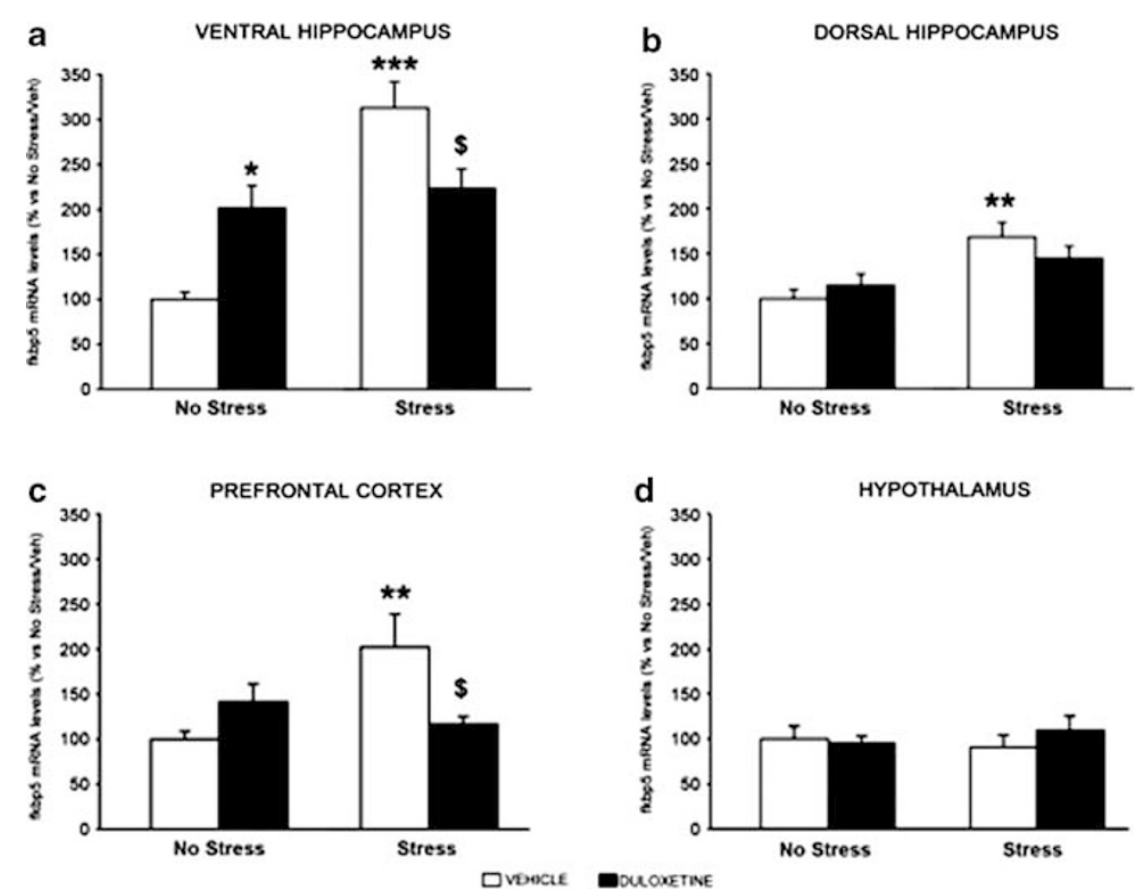

Figure 3 Modulation of Fkbp5 expression in the rat brain following chronic stress and antidepressant treatment. The mRNA levels of Fkbp5 were measured in ventral hippocampus (a), dorsal hippocampus (b), prefrontal cortex (c), and hypothalamus (d) of non-stressed and chronically stressed rats, treated for $2 \mathrm{I}$ days with vehicle or duloxetine and killed $24 \mathrm{~h}$ after the last stress. The data, expressed as a percentage of No Stress/Vehicle (set at I00\%), are the mean \pm SEM of $6-12$ independent determinations. $* P<0.05$, ${ }^{*} * P<0.01$, ${ }^{*} * * P<0.01$ vs No Stress/Vehicle; ${ }^{\$} P<0.05$ vs Stress/Vehicle (two-way ANOVA with SCPHT).

a
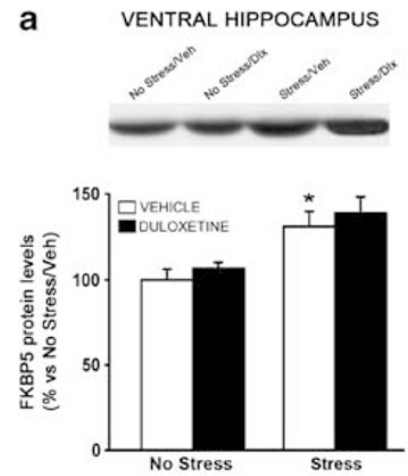

b
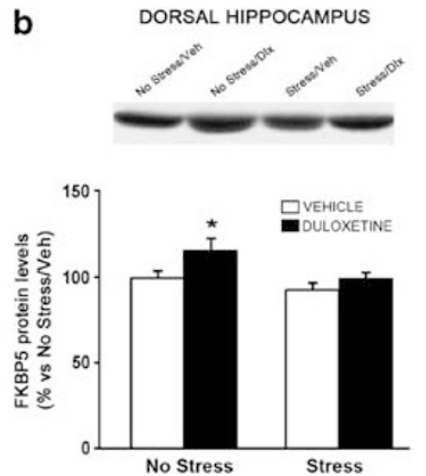

C
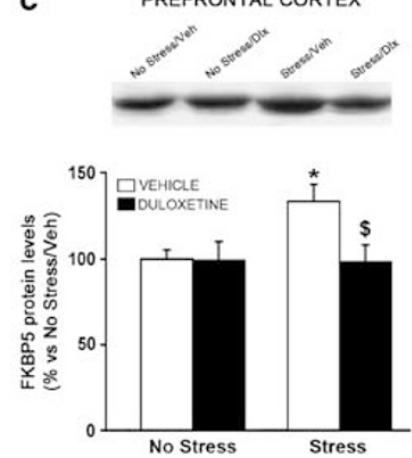

Figure 4 Modulation of FKBP5 protein levels by CMS and antidepressant treatment in the rat brain. The protein levels of FKBP5 were measured in the cytosolic fraction of ventral hippocampus (a), dorsal hippocampus (b), and prefrontal cortex (c) of non-stressed and chronically stressed rats, treated for 21 days with vehicle or duloxetine and killed $24 \mathrm{~h}$ after the last stress. The data, expressed as a percentage of No Stress/Vehicle (set at I00\%), are the mean \pm SEM of 5-7 independent determinations. ${ }^{*} P<0.05$ vs No Stress/Vehicle; ${ }^{\$} P<0.05$ vs Stress/Vehicle (two-way ANOVA with SCPHT).

$P>0.05, v s$ No Stress/Veh animals). Finally in the hypothalamus, Fkbp5 expression was not influenced by stress $\left(\mathrm{F}_{1,24}=0.82, P>0.05\right)$ or by drug treatment $\left(\mathrm{F}_{1,24}=1.18\right.$, $P>0.05)$ (Figure 3d).

Based on these data, we decided to examine the protein levels of FKBP5 in the cytosolic fraction of ventral, dorsal hippocampus, and prefrontal cortex. The protein levels of this immunophilin mirrored only in part the changes found in gene expression. As shown in Figure $4 \mathrm{a}$, in the ventral hippocampus we found a significant stress effect $\left(\mathrm{F}_{1,25}=16.19, \quad P<0.001\right)$ : CMS increased FKBP5 levels ( $+31 \%, P<0.05$ vs No Stress/Veh animals), an effect that was not modulated by chronic duloxetine treatment. In the dorsal hippocampus (Figure 4b), we found no effect of chronic stress but a significant treatment effect $\left(\mathrm{F}_{1,25}=5.74\right.$,
$P<0.05)$, which was due to the significant increase in FKBP5 protein $(+16 \%, P<0.05$ vs No Stress/Veh animals) following duloxetine administration to control rats. In the prefrontal cortex (Figure 4c), CMS significantly increased FKBP5 protein levels $(+33 \%, P<0.05$ vs No Stress/Veh animals), whereas duloxetine treatment reversed the upregulation found in stressed rats $(-27 \%, P<0.05$, vs No Stress/Veh animals) without affecting the chaperone levels in control animals.

\section{Modulation of GR mRNA and Protein Levels}

We next investigated the expression of the GR. With respect to the transcriptional levels of the gene coding for GR, $\mathrm{Nr} 3 \mathrm{c}$, we did not find any effect of CMS in the ventral 

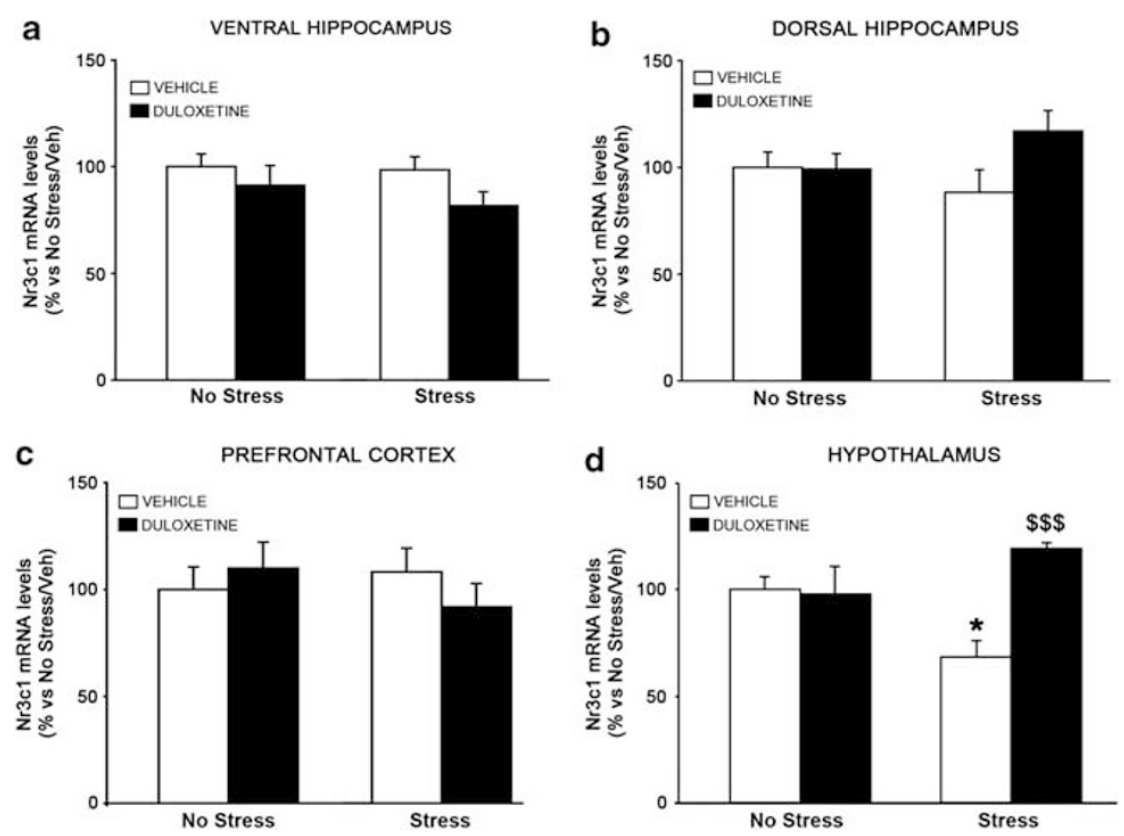

Figure 5 Modulation of $\mathrm{Nr} 3 \mathrm{cl}$ expression in the rat brain following chronic stress and antidepressant treatment. The mRNA levels of $\mathrm{Nr} 3 \mathrm{cl}$ were measured in ventral hippocampus (a), dorsal hippocampus (b), prefrontal cortex (c), and hypothalamus (d) of non-stressed and chronically stressed rats, treated for $2 \mathrm{I}$ days with vehicle or duloxetine and killed $24 \mathrm{~h}$ after the last stress. The data, expressed as a percentage of No Stress/Vehicle (set at I00\%), are the mean \pm SEM of $6-12$ independent determinations. $* P<0.05$ vs No Stress/Vehicle; ${ }^{\$ \$} P<0.00$ I vs Stress/Vehicle (two-way ANOVA with SCPHT).

hippocampus $\left(\mathrm{F}_{1,48}=0.61, P>0.05\right)$ (Figure 5a), dorsal hippocampus $\left(\mathrm{F}_{1,46}=0.12, P>0.05\right)$ (Figure $5 \mathrm{~b}$ ), and prefrontal cortex $\left(\mathrm{F}_{1,47}=0.19, P>0.05\right)$ (Figure $\left.5 \mathrm{c}\right)$. Furthermore, $\mathrm{Nr} 3 \mathrm{cl}$ expression was not affected by chronic duloxetine treatment, in the these brain regions $\left(\mathrm{F}_{1,48}=\right.$ $3.29, P>0.05$ for ventral hippocampus; $\mathrm{F}_{1,46}=2.59, P>0.05$ for dorsal hippocampus; $F_{1,47}=0.08, P>0.05$ for prefrontal cortex) (Figure $5 \mathrm{a}-\mathrm{c}$ ). On the contrary in the hypothalamus, $\mathrm{Nr} 3 \mathrm{c} 1 \mathrm{mRNA}$ levels were significantly regulated by treatment $\left(\mathrm{F}_{1,24}=8.55, \quad P<0.01\right)$ with a significant stress $\times$ treatment interaction $\left(\mathrm{F}_{1,24}=9.86, P<0.01\right)$ (Figure $5 \mathrm{~d}$ ). $\mathrm{Nr} 3 \mathrm{c} 1$ expression was significantly reduced following CMS ( $-31 \%, P<0.05$ vs No Stress/Veh animals). Duloxetine did not affect $\mathrm{Nr} 3 \mathrm{cl}$ mRNA levels when given to control rats ( $-2 \%, P>0.05$ vs No Stress/Veh animals), but increased its expression when administered to CMS rats $(+74 \%$, $P<0.001$ vs Stress/Veh animals).

We next examined the modulation of GR protein, by investigating its levels in the nuclear $v s$ the cytosolic compartments. Figure 6 shows prototypical western blot analyses for GR levels in the cytosolic and nuclear fractions. As shown in Figure 7a, in the cytosolic fraction of ventral hippocampus we found a significant effect of chronic stress $\left(\mathrm{F}_{1,22}=14.61, P<0.01\right)$ : GR levels were significantly increased following CMS ( $+32 \%, P<0.05$ vs No Stress/Veh animals), an effect that was not influenced by the antidepressant administration. In contrast, the expression of GR in the nucleus was not modulated by stress or duloxetine $\left(\mathrm{F}_{1,24}=0.85, P>0.05\right.$ and $\mathrm{F}_{1,24}=0.05, P>0.05$, respectively $)$ (Figure 7d). In the cytosolic fraction from the dorsal hippocampus, GR levels were not affected either by CMS $\left(F_{1,25}=0.78, P>0.05\right)$ or by duloxetine $\left(F_{1,25}=0.07\right.$, $P>0.05$ ) (Figure $7 \mathrm{~b}$ ). Nuclear analysis (Figure $7 \mathrm{e}$ ), on the contrary, shows a significant stress $\times$ treatment interaction
$\left(\mathrm{F}_{1,25}=13.92, P<0.01\right)$, with stress inducing a small, but not significant, increase of GR $(+35 \%, P>0.05$, vs Stress/Veh animals), and duloxetine reducing GR levels when given to stressed animals ( $-45 \%, P<0.05$ vs Stress/Veh animals), but not in sham rats $(+28 \%, P>0.05$, vs No Stress/Veh animals). Finally, when analyzing the modulation of GR in the cytosolic fraction of the prefrontal cortex (Figure 7c), we found significant stress $\left(\mathrm{F}_{1,25}=5.97, P<0.05\right)$ and treatment $\left(\mathrm{F}_{1,25}=42.52, P<0.001\right)$ effects as well as a significant stress $\times$ treatment interaction $\left(\mathrm{F}_{1,25}=11.34, P<0.01\right)$. Indeed, CMS increased the receptor levels $(+53 \%, P<0.001$ $v s$ No Stress/Veh animals), while duloxetine reduced it when given to the stressed rats $(-65 \%, P<0.001$ vs Stress/ Veh animals). In the nucleus (Figure 7f), we found a significant stress $\times$ treatment interaction $\left(\mathrm{F}_{1,25}=11.34\right.$, $P<0.01)$. In fact CMS induced a small, but not significant, increase in GR ( $+35 \%, P>0.05$ vs No Stress/Veh animals), while chronic duloxetine decreased its levels, when given to stressed rats $(-59 \%, P<0.05$ vs Stress/Veh animals).

\section{Analysis of GR Phosphorylation}

To gain further insight into the mechanism that may regulate GR trafficking, we investigated the pattern of GR phosphorylation in the nuclear and cytosolic compartments of the prefrontal cortex, the brain region where major changes occurred on GR levels in two compartments (see Figure 7). We focused our analysis on the three phosphorylation sites within its N-terminal (Ser224, Ser232, Ser246), which regulate GR trafficking and GR-dependent gene transcription (Wang et al, 2002; Galliher-Beckley et al, 2008; Takabe et al, 2008) and that have recently been shown to be modulated by GC hormones and antidepressants in human hippocampal stem cells (Anacker et al, 2011). Specifically, 
a

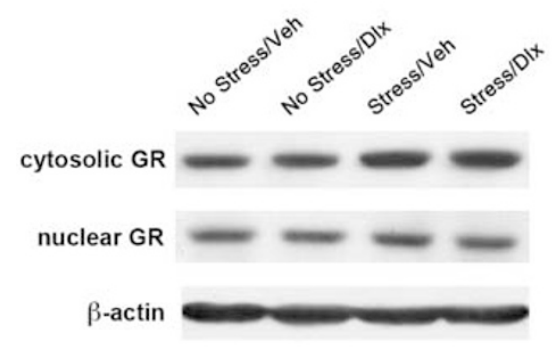

C

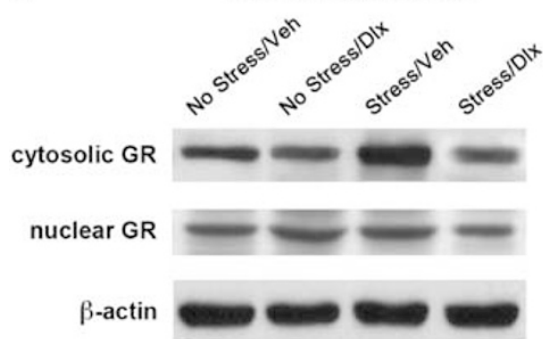

b

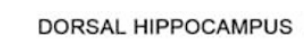

cytosolic GR

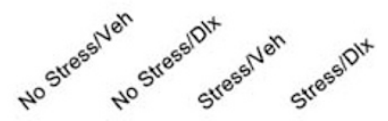

nuclear GR

$\beta$-actin

d

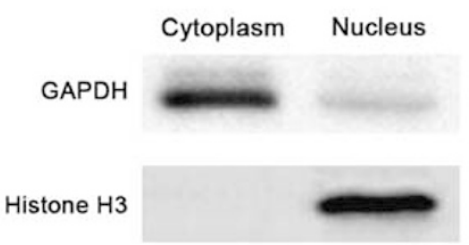

Figure 6 Representative western blot analyses of GR protein expression (cytosolic or nuclear compartments) and $\beta$-actin, used as internal standard, are shown for ventral hippocampus (a), dorsal hippocampus (b), and prefrontal cortex (c). (d) The purity of subcellular fractions was assayed by anti-GAPDH or anti-Histone $\mathrm{H} 3$ antibodies for the cytoplasmic or nuclear compartments, respectively. Veh: vehicle, dlx: duloxetine. Experimental conditions are described in Materials and methods.
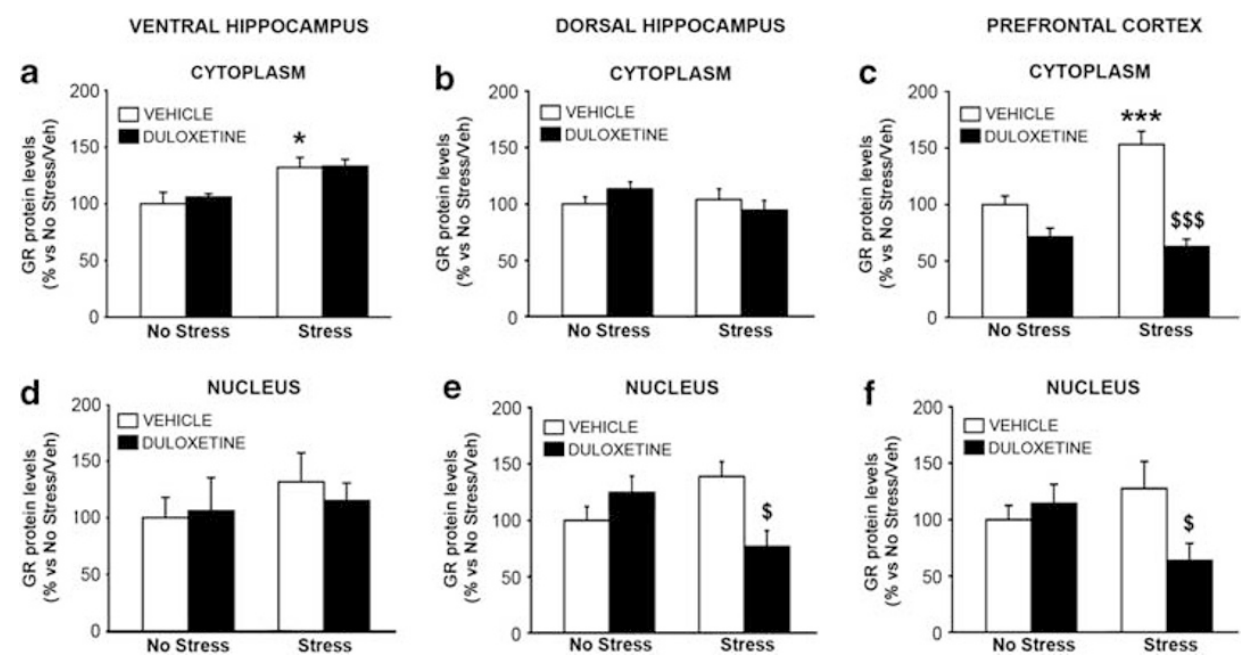

Figure 7 Modulation of GR protein levels in the rat brain following CMS and antidepressant treatment. The protein levels of GR were measured in the cytoplasm ( $a-c)$ and in the nucleus ( $d-f)$ of ventral hippocampus $(a, d)$, dorsal hippocampus (b, e), and prefrontal cortex (c, f) of non-stressed and chronically stressed rats, treated for $2 \mathrm{I}$ days with vehicle or duloxetine and killed $24 \mathrm{~h}$ after the last stress. The data, expressed as a percentage of No Stress/Vehicle (set at $100 \%$ ), are the mean \pm SEM of 5-7 independent determinations. ${ }^{*} P<0.05$, ${ }^{*} * * P<0.00$ I vs No Stress/Vehicle; ${ }^{\$} P<0.05$, ${ }^{\$ \$} P<0.00$ I vs Stress/Vehicle (two-way ANOVA with SCPHT).

we focused on the pSer224/total GR and pSer232/total GR ratios in the cytoplasm, because phosphorylation at these sites may lead to nuclear translocation of the receptor, and pSer246/total GR ratio in the nuclear compartment, because this may be important for the nuclear export of the receptor.

With respect to the pSer224/total GR levels in the cytosol (Figure 8a), we found significant stress $\left(\mathrm{F}_{1,23}=\right.$ 11.27, $P<0.01)$ and treatment $\left(\mathrm{F}_{1,23}=254.74, P<0.001\right)$ effects. Phosphorylation on Ser224 was slightly, although not significantly, decreased in stressed animals $(-17 \%$, $P>0.05$ vs No Stress/Veh animals). Conversely, chronic duloxetine treatment elicited a significant increase in pSer224 GR levels in stressed $(+132 \%, P<0.001$ vs Stress/Veh animals), as well as in sham animals $(+125 \%$, $P>0.001$ vs No Stress/Veh animals). When considering cytosolic pSer232/GR ratio (Figure 8b) we found that the phosphorylation was also significantly regulated by stress $\left(\mathrm{F}_{1,24}=11.97, P<0.01\right)$ and treatment $\left(\mathrm{F}_{1,24}=31.74\right.$, $P<0.001)$. CMS significantly decreased the ratio of pSer232/ total GR levels ( $-36 \%, P<0.01$ vs No Stress/Veh animals), while duloxetine increased this ratio $(+89 \%, P<0.001$ vs No Stress/Veh animals). The antidepressant alone was also 


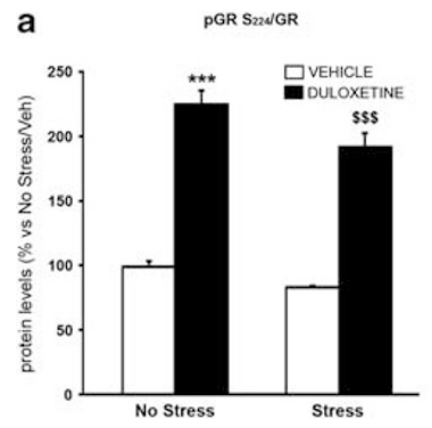

b

PGR S232/GR

C
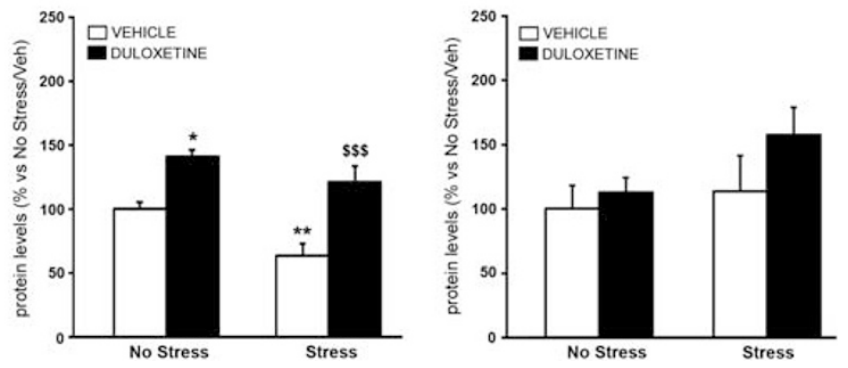

Figure 8 Modulation of three GR phosphorylation at three different sites in the rat prefrontal cortex following CMS and/or antidepressant treatment. The levels of pSer224 (a) and pSer232 (b) were measured in the cytosolic fraction, whereas pSer246 (c) was measured in the nuclear compartment of prefrontal cortex from non-stressed or chronically stressed rats, treated for 21 days with vehicle or duloxetine and killed $24 \mathrm{~h}$ after the last stress. The data represent the ratio between phosphorylated and total GR levels. Results, expressed as a percentage of No Stress/Vehicle (set at I00\%), are the mean \pm SEM of 5-7 independent determinations. $* P<0.05$, $* * P<0.01$, **** $P<0.00$ I vs No Stress/Vehicle; ${ }^{\$}{ }^{\$} P<0.00$ I vs Stress/Vehicle (two-way ANOVA with SCPHT).
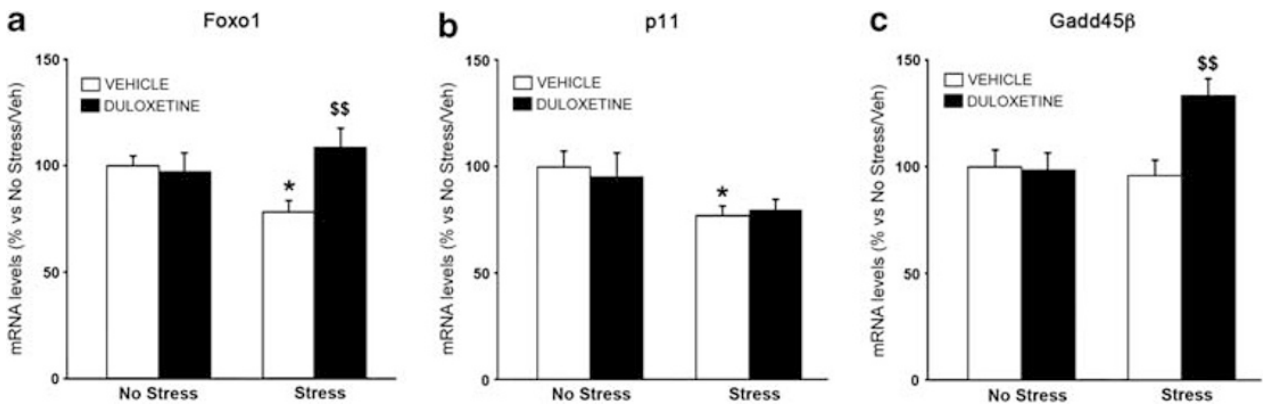

Figure 9 Modulation of GR regulated gene expression in the rat prefrontal cortex following CMS and/or antidepressant treatment. The mRNA levels of forkhead box OI (FOXOI) (a), SIO0 calcium binding protein AIO (pII) (b), and growth arrest and DNA damage-inducible, beta (Gadd45 $\beta$ ) (c) were measured in the prefrontal cortex of non-stressed and chronically stressed rats, treated with vehicle or duloxetine and killed $24 \mathrm{~h}$ after the last stress. The data, expressed as a percentage of No Stress/Vehicle (set at 100\%), are the mean \pm SEM of 10-12 independent determinations. *P<0.05 vs No Stress/ Vehicle; ${ }^{\$ \$} P<0.01$ vs Stress/Vehicle (two-way ANOVA with SCPHT).

able to increase the phosphorylation on pSer232 in controls animals ( $+41 \%, P<0.05$ vs Stress/Veh animals).

Last, we analyzed pSer246/GR ratio GR phosphorylation on Ser246 in the nuclear compartment. Differently from pSer224 and pSer232, we found that pSer246/GR ratio (Figure 8c) was not affected by CMS $\left(\mathrm{F}_{1,22}=1.50, P>0.05\right)$ or by treatment $\left(\mathrm{F}_{1,23}=1.43, P>0.05\right)$, which is in line with recent observations on the effects of GC hormones and antidepressants on this phosphorylation site (Anacker et al, 2011).

\section{Modulation of GR Responsive Genes}

Considering that, within the prefrontal cortex, CMS and duloxetine treatment were able to affect GR phosphorylation that holds implications for its transcriptional activity, we analyzed three representative GR responsive genes, forkhead box O1 (Foxo1), S100 calcium binding protein A10 (p11) and growth arrest and DNA-damage-inducible, beta $(\operatorname{Gadd} 45 \beta)$, which have been recently implicated in antidepressant drug action (Egeland et al, 2010; Polter et al, 2009; Svenningsson et al, 2006).

As shown in Figure 9a, with respect to Foxol mRNA levels, we found a significant stress $\times$ treatment interaction $\left(F_{1,46}=5.86, P<0.05\right)$. Foxol expression was significantly reduced following CMS $(-22 \%, P<0.05$ vs No Stress/Veh animals), an effect that was normalized by chronic duloxetine treatment ( $+40 \%, P<0.01$ vs Stress/Veh animals). Regarding p11 (Figure 9b) we found a significant effect of chronic stress $\left(F_{1,46}=7.64, P<0.01\right)$. Indeed, p11 mRNA levels were significantly reduced in rats exposed to CMS $(-23 \%, P<0.05$, vs No Stress/Veh animals), an effect that, however, was not influenced by chronic duloxetine administration. Last, we analyzed the modulation of Gadd $45 \beta$ (Figure 9c), and we found a significant treatment effect $\left(\mathrm{F}_{1,47}=5.93, P<0.05\right)$ with a significant stress $\times$ treatment interaction $\left(\mathrm{F}_{1,47}=6.99, P<0.05\right)$. Differently from the previous two genes, CMS did not influence Gadd $45 \beta$ gene expression $(-4 \%, P>0.05)$, whereas chronic DLX treatment was able to increase Gadd $45 \beta$ mRNA levels in stressed rats $(+40 \%, P<0.01)$, without affecting its expression in sham animals $(-2 \%, P>0.05)$.

\section{DISCUSSION}

In this study, we provide evidence that animals exposed to CMS show alteration of the system FKBP5-GR that is important for HPA axis function and GR activity. The changes produced by CMS, which reproduces a depressive-like 
phenotype, are suggestive of reduced translocation and function of GR in the ventral and dorsal hippocampus as well as in the prefrontal cortex, and we demonstrate that chronic treatment with the antidepressant duloxetine is able to normalize some of these alterations, primarily in the prefrontal cortex.

FKBP5 is a co-chaperone of hsp90, which regulates GR sensitivity, lowering the affinity for its ligand and reducing the efficiency of the nuclear translocation. We found increased expression of FKBP5 after CMS, which may inhibit GR translocation to the nuclear compartment. This effect was found in most of the brain regions investigated, although larger changes were observed in ventral hippocampus and prefrontal cortex. While we did not find similar changes in the hypothalamus, in this region CMS significantly reduced GR mRNA levels. A likely consequence of these changes may be an impaired HPA axis feedback inhibition as well as GR resistance. Indeed, we show that increased FKBP5 expression in CMS rats is associated with increased GR levels in the cytosolic compartment of ventral hippocampus and prefrontal cortex, which may be suggestive of an impaired ability of the receptor to translocate to the nucleus and activate GR-dependent transcriptional mechanisms. In line with this possibility, polymorphisms of the human FKBP5 gene that are associated with enhanced expression of the co-chaperone protein affect GR sensitivity, leading to increased GR resistance and decreased efficiency of the negative feedback control, resulting in a prolongation of the hormonal response following stress exposure (Binder et al, 2008; Ising et al, 2008) and, in general, to HPA axis hyperactivity because of an impaired feedback inhibition by GCs. The same alleles are overrepresented in individuals with major depression, bipolar disorder and post-traumatic stress disorder (Binder, 2009) and increased FKBP5 mRNA and protein expression have been reported in post-mortem frontal cortex from individuals suffering from major depression (Tatro et al, 2009). Moreover, two studies have shown that the alleles associated with enhanced induction of FKBP5 are associated with increased risk to develop PTSD or related symptoms (Koenen et al, 2005).

Our results show that the ventral hippocampus, which participates in emotional regulation and anxiety-related behaviors, is more affected by stress exposure when compared with the dorsal part, which is preferentially involved in learning and memory. In fact, we found a selective increase in FKBP5 and GR protein levels in the ventral hippocampus but not in the dorsal subregion. These results are in accordance with recent data demonstrating that chronic stress exposure leads to reduced cell proliferation and neurogenesis preferentially in the ventral hippocampus (Tanti et al, 2012).

We also demonstrate that impaired function of GR following chronic stress may be sustained by changes in its phosphorylation, also in agreement with recent work, from some of the authors of the present paper, in cultured hippocampal stem cells (Anacker et al, 2011).

While cell type-specific mechanism are important for cellular trafficking of GRs (Fitzsimons et al, 2008), the state of receptor phosphorylation may have a role in controlling activation, subcellular localization, gene transcription, and turnover of GRs (Chen et al, 2008; Ismaili and Garabedian, 2004). The receptor can be phosphorylated as a conse- quence of ligand binding (Ismaili and Garabedian, 2004; Rogatsky et al, 1998), but it may also occur through the activation of different signaling pathways, including protein kinase A (PKA) (Anacker et al, 2011; Rangarajan et al, 1992), cyclin-dependent kinase (CDK) (Krstic et al, 1997), glycogen synthase kinase-3 (GSK3) and c-Jun N-terminal kinases (JNK) (Rogatsky et al, 1998), which may cooperate to modulate GR sensitivity and function. We show that phosphorylation of GR on Ser232, and to less extent on Ser224, is significantly decreased as a consequence of CMS. Previous studies have shown that GR phosphorylation at these sites are induced by PKA (Anacker et al, 2011; Rangarajan et al, 1992) and may regulate GR trafficking to the nucleus, suggesting that reduced phosphorylation on Ser232 may also contribute to impaired GR function as a consequence of stress exposure.

Of note is that these findings are in apparent contrast from those recently obtained by some of us in an in vitro study using human neuronal stem cells (Anacker et al, 2011), where an increase in GR phosphorylation at the Ser232 site has been described following incubation of cells with the GR agonist, dexamethasone, for 1-12 h. It may be inferred that under acute conditions GCs may promote GR phosphorylation leading to nuclear translocation, which may be important for coping mechanisms, whereas chronic stress may lead to an impairment of GR function also through a decreased phosphorylation on Ser224 and Ser232.

While GRs have a key role in HPA axis regulation, they have widespread activity by regulating the transcription of several genes that may also have a role in mood disorders, such as the neurotrophin BDNF. We have recently shown that GR-deficient mice $\left(\mathrm{GR}^{+/-}\right)$, a genetic model of predisposition to depression (Ridder et al, 2005), show altered modulation of BDNF expression in response to an acute stress (Molteni et al, 2010) and impaired adult hippocampal neurogenesis, which is further reduced after stress exposure (Kronenberg et al, 2009). These data implicate that impaired GR function as a consequence of chronic stress, may lead to abnormalities of neurotrophic and neuroplastic mechanisms, which may contribute to functional deterioration in stress-related disorders (Anacker et al, 2011; Calabrese et al, 2011). Moreover, GR compromised mice show profound alterations for the expression and function of 5-HT receptors (Hensler et al, 2010; Trajkovska et al, 2009), providing further support to the functional link existing between HPA axis and serotonergic system in the context of mood disorders.

To demonstrate that increased FKBP5 levels, accumulation of cytosolic GR, and alterations of its phosphorylation pattern are associated with the reduced GR function in the prefrontal cortex, we analyzed the expression of the GR responsive genes FOXO1, p11, and GADD $45 \beta$. We show that FOXO1 and p11 gene expression is reduced in stressed animals, while GADD45 $\beta$ is not affected. FOXO1 is a transcription factor involved in cell survival, differentiation, and resistance to oxidative stress (Nemoto and Finkel, 2002). It may be inferred that a reduction of its expression impairs the maintenance of cellular homeostasis under challenging conditions. With respect to p11, our data are in agreement with previous results showing that its expression is decreased in brain tissue of depressed individuals (Anisman et al, 2008; Svenningsson et al, 2006) as well as 
in an animal model of depression (Svenningsson et al, 2006), and that the p11 knockout (KO) mice have a depression-like phenotype (Svenningsson et al, 2006; Warner-Schmidt et al, 2009).

Finally, we provide evidence that chronic antidepressant treatment is able to normalize, at least in part, the alterations caused by the CMS exposure, in an areadependent manner. The SNRI duloxetine reduces the mRNA levels of FKBP5 in the ventral hippocampus, and completely normalizes the alteration of both FKBP5 mRNA and protein, as well as cytosolic GR levels in the prefrontal cortex. Several in vitro and in vivo studies suggest a close interaction between antidepressants and the GR system. Treatment with tricyclic antidepressants increases GR binding affinity and GR mRNA expression in rat hypothalamic and hippocampal neurons, suggesting that, in line with our results, antidepressants may enhance GC sensitivity and function, specifically in the brain, and thereby may restore GR-mediated feedback inhibition on the HPA axis (Okugawa et al, 1999; Peiffer et al, 1991). Furthermore, SSRI and tricyclic antidepressants increase nuclear translocation of GRs and also facilitate GR-mediated gene transcription (Anacker et al, 2011; Pariante et al, 2003). Importantly, GR phosphorylation at Ser224 and Ser232 critically controls GR-dependent gene transcription by GC hormones and antidepressants (Anacker et al, 2011).

Our results are remarkably consistent with the in vitro data and suggest that different antidepressants may elicit significant effects on GR-HPA axis function. We show, for the first time in an animal model, that one novel mechanism through which antidepressants may regulate GR function is the modulation of its phosphorylation. Specifically, our data demonstrate that the phosphorylation on pSer224 and pSer232 is significantly upregulated by duloxetine, as previously shown for sertraline in human hippocampal stem cells (Anacker et al, 2011). This effect normalizes the changes produced by CMS, particularly when considering pSer232 and, more in general, may represent a mechanism through which drug treatment may regulate different signaling pathways that will ultimately affect GR function and GRdependent changes in HPA axis function. Accordingly, we also show that chronic duloxetine treatment upregulates the expression of two GR responsive genes FOXO1 and Gadd $45 \beta$, selectively in CMS rats. While these changes restore the expression of FOXO1 reduced by CMS (see above), the increase in Gadd $45 \beta$ expression may be of interest considering that the gene is involved in DNA demethylation of several promoters including BDNF ( $\mathrm{Ma}$ et al, 2009), which is important for neuroplasticity and antidepressant activity (Calabrese et al, 2010; Schmidt et al, 2011). These results strengthen the link between the modulation of HPA axis activity and neuroadaptive mechanisms exerted by antidepressant drugs, although neuroprotective effects may be mediated and sustained by different mechanisms, since we have shown that the SNRI duloxetine may be more effective than fluoxetine in elevating BDNF expression (Calabrese et al, 2007, 2010; Molteni et al, 2009; Shirayama et al, 2002), whereas chronic treatment with fluoxetine, but not duloxetine, exert significant effects on hippocampal neurogenesis (Marlatt et al, 2010).

In conclusion, our data suggest that chronic stress produces alterations in the mechanisms controlling GR translocation and that the pharmacological modulation of these mechanisms may contribute to normalize the complex array of dysfunctions associated with stress-related disorders.

\section{ACKNOWLEDGEMENTS}

This publication was made possible by grants to MA Riva from Regione Lombardia (Accordo Quadro 2010) and by a liberal contribution from Eli Lilly Italia, as well as from grants from the Commission of European Communities 7th Framework Programme (Collaborative Project Grant Agreement $n$ 22963, Mood Inflame) and the Medical Research Council, UK (G108/603) to C Pariante, and from the National Institute for Health Research (NIHR) Mental Health Biomedical Research Centre at South London and Maudsley NHS Foundation Trust and King's College London, to C Anacker. We would like to thank Dr Michael J Garabedian for the GR phospho-specific antibodies.

\section{DISCLOSURE}

GR has received compensation as speaker/consultant for Eli Lilly, InnovaPharma, and Servier. CMP has received speakers' fees from Eli Lilly, the company producing duloxetine, as well as from other pharmaceutical companies producing antidepressants. MAR has received compensation as speaker/consultant for AstraZeneca, Bristol-Myers Squibb, Eli Lilly, Servier, and Takeda.

\section{REFERENCES}

Anacker C, Zunszain PA, Cattaneo A, Carvalho LA, Garabedian MJ, Thuret $S$ et al (2011). Antidepressants increase human hippocampal neurogenesis by activating the glucocorticoid receptor. Mol Psychiatry 16: 738-750.

Anisman H, Du L, Palkovits M, Faludi G, Kovacs GG, SzontaghKishazi $\mathrm{P}$ et al (2008). Serotonin receptor subtype and p11 mRNA expression in stress-relevant brain regions of suicide and control subjects. J Psychiatry Neurosci 33: 131-141.

Binder EB (2009). The role of FKBP5, a co-chaperone of the glucocorticoid receptor in the pathogenesis and therapy of affective and anxiety disorders. Psychoneuroendocrinology 34(Suppl 1): S186-S195.

Binder EB, Bradley RG, Liu W, Epstein MP, Deveau TC, KB Mercer et al (2008). Association of FKBP5 polymorphisms and childhood abuse with risk of posttraumatic stress disorder symptoms in adults. JAMA 299: 1291-1305.

Calabrese F, Guidotti G, Molteni R, Racagni G, Mancini M, Riva MA (2012). Stress-induced changes of hippocampal NMDA receptors: modulation by duloxetine treatment. PLOS ONE 7: e37916.

Calabrese F, Molteni R, Cattaneo A, Macchi F, Racagni G, Gennarelli M et al (2010). Long-Term duloxetine treatment normalizes altered brain-derived neurotrophic factor expression in serotonin transporter knockout rats through the modulation of specific neurotrophin isoforms. Mol Pharmacol 77: 846-853.

Calabrese F, Molteni R, Maj PF, Cattaneo A, Gennarelli M, Racagni $\mathrm{G}$ et al (2007). Chronic duloxetine treatment induces specific changes in the expression of BDNF transcripts and in the subcellular localization of the neurotrophin protein. Neuropsychopharmacology 32: 2351-2359. 
Calabrese F, Molteni R, Riva MA (2011). Antistress properties of antidepressant drugs and their clinical implications. Pharmacol Ther 132: 39-56.

Chen W, Dang T, Blind RD, Wang Z, Cavasotto CN, Hittelman AB et al (2008). Glucocorticoid receptor phosphorylation differentially affects target gene expression. Mol Endocrinol 22: 1754-1766.

de Kloet ER, Joels M, Holsboer F (2005). Stress and the brain: from adaptation to disease. Nat Rev Neurosci 6: 463-475.

Egeland M, Warner-Schmidt J, Greengard P, Svenningsson P (2010). Neurogenic effects of fluoxetine are attenuated in p11 (S100A10) knockout mice. Biol Psychiatry 67: 1048-1056.

Fitzsimons CP, Ahmed S, Wittevrongel CF, Schouten TG, Dijkmans TF, Scheenen WJ et al (2008). The microtubuleassociated protein doublecortin-like regulates the transport of the glucocorticoid receptor in neuronal progenitor cells. Mol Endocrinol 22: 248-262.

Frampton JE, Plosker GL (2007). Duloxetine: a review of its use in the treatment of major depressive disorder. CNS Drugs 21: 581-609.

Galliher-Beckley AJ, Williams JG, Collins JB, Cidlowski JA (2008). Glycogen synthase kinase 3beta-mediated serine phosphorylation of the human glucocorticoid receptor redirects gene expression profiles. Mol Cell Biol 28: 7309-7322.

Guidotti G, Calabrese F, Auletta F, Olivier J, Racagni G, Homberg J et al (2012). Developmental influence of the serotonin transporter on the expression of npas4 and GABAergic markers: modulation by antidepressant treatment. Neuropsychopharmacology 37: 746-758.

Hensler JG, Vogt MA, Gass P (2010). Regulation of cortical and hippocampal 5-HT(1A) receptor function by corticosterone in GR + /- mice. Psychoneuroendocrinology 35: 469-474.

Holsboer F (2000). The corticosteroid receptor hypothesis of depression. Neuropsychopharmacology 23: 477-501.

Ising M, Depping AM, Siebertz A, Lucae S, Unschuld PG, Kloiber S et al (2008). Polymorphisms in the FKBP5 gene region modulate recovery from psychosocial stress in healthy controls. Eur $J$ Neurosci 28: 389-398.

Ismaili N, Garabedian MJ (2004). Modulation of glucocorticoid receptor function via phosphorylation. Ann NY Acad Sci 1024: 86-101.

Koenen KC, Saxe G, Purcell S, Smoller JW, Bartholomew D, Miller A et al (2005). Polymorphisms in FKBP5 are associated with peritraumatic dissociation in medically injured children. $\mathrm{Mol}$ Psychiatry 10: 1058-1059.

Kronenberg G, Kirste I, Inta D, Chourbaji S, Heuser I, Endres M et al (2009). Reduced hippocampal neurogenesis in the GR( + /-) genetic mouse model of depression. Eur Arch Psychiatry Clin Neurosci 259: 499-504.

Krstic MD, Rogatsky I, Yamamoto KR, Garabedian MJ (1997). Mitogen-activated and cyclin-dependent protein kinases selectively and differentially modulate transcriptional enhancement by the glucocorticoid receptor. Mol Cell Biol 17: 3947-3954.

Ma DK, Jang MH, Guo JU, Kitabatake Y, Chang ML, PowAnpongkul N et al (2009). Neuronal activity-induced Gadd45b promotes epigenetic DNA demethylation and adult neurogenesis. Science 323: 1074-1077.

Marlatt MW, Lucassen PJ, van Praag H (2010). Comparison of neurogenic effects of fluoxetine, duloxetine and running in mice. Brain Res 1341: 93-99.

McEwen BS, Gould EA, Sakai RR (1992). The vulnerability of the hippocampus to protective and destructive effects of glucocorticoids in relation to stress. Br J Psychiatry Suppl 15: 18-23.

Molteni R, Calabrese F, Cattaneo A, Mancini M, Gennarelli M, Racagni $G$ et al (2009). Acute stress responsiveness of the neurotrophin BDNF in the rat hippocampus is modulated by chronic treatment with the antidepressant duloxetine. Neuropsychopharmacology 34: 1523-1532.
Molteni R, Calabrese F, Chourbaji S, Brandwein C, Racagni G, Gass $P$ et al (2010). Depression-prone mice with reduced glucocorticoid receptor expression display an altered stress-dependent regulation of brain-derived neurotrophic factor and activityregulated cytoskeleton-associated protein. J Psychopharmacol 24: 595-603.

Nemoto S, Finkel T (2002). Redox regulation of forkhead proteins through a p66shc-dependent signaling pathway. Science 295: $2450-2452$.

Okugawa G, Omori K, Suzukawa J, Fujiseki Y, Kinoshita T, Inagaki C (1999). Long-term treatment with antidepressants increases glucocorticoid receptor binding and gene expression in cultured rat hippocampal neurones. J Neuroendocrinol 11: 887-895.

Pariante CM (2006). The glucocorticoid receptor: part of the solution or part of the problem? J Psychopharmacol 20: 79-84.

Pariante CM, Kim RB, Makoff A, Kerwin RW (2003). Antidepressant fluoxetine enhances glucocorticoid receptor function in vitro by modulating membrane steroid transporters. $\mathrm{Br} J$ Pharmacol 139: 1111-1118.

Pariante CM, Lightman SL (2008). The HPA axis in major depression: classical theories and new developments. Trends Neurosci 31: 464-468.

Paxinos G, Watson C (1996). The Rat Brain in Stereotaxis Coordinates. Academic Press: New York.

Peiffer A, Veilleux S, Barden N (1991). Antidepressant and other centrally acting drugs regulate glucocorticoid receptor messenger RNA levels in rat brain. Psychoneuroendocrinology 16: 505-515.

Pittenger C, Duman RS (2008). Stress, depression, and neuroplasticity: a convergence of mechanisms. Neuropsychopharmacology 33: 88-109.

Polter A, Yang S, Zmijewska AA, van Groen T, Paik JH, Depinho RA et al (2009). Forkhead box, class O transcription factors in brain: regulation and behavioral manifestation. Biol Psychiatry 65: $150-159$

Rangarajan PN, Umesono K, Evans RM (1992). Modulation of glucocorticoid receptor function by protein kinase A Mol Endocrinol 6: 1451-1457.

Ridder S, Chourbaji S, Hellweg R, Urani A, Zacher C, Schmid W et al (2005). Mice with genetically altered glucocorticoid receptor expression show altered sensitivity for stress-induced depressive reactions. J Neurosci 25: 6243-6250.

Rogatsky I, Waase CL, Garabedian MJ (1998). Phosphorylation and inhibition of rat glucocorticoid receptor transcriptional activation by glycogen synthase kinase-3 (GSK-3). Species-specific differences between human and rat glucocorticoid receptor signaling as revealed through GSK-3 phosphorylation. J Biol Chem 273: 14315-14321.

Schmidt HD, Shelton RC, Duman RS (2011). Functional biomarkers of depression: diagnosis, treatment, and pathophysiology. Neuropsychopharmacology 36: 2375-2394.

Shirayama Y, Chen AC, Nakagawa S, Russell DS, Duman RS (2002). Brain-derived neurotrophic factor produces antidepressant effects in behavioral models of depression. J Neurosci 22: 3251-3261.

Svenningsson P, Chergui K, Rachleff I, Flajolet M, Zhang X, El Yacoubi $M$ et al (2006). Alterations in 5-HT1B receptor function by 111 in depression-like states. Science 311: 77-80.

Takabe S, Mochizuki K, Goda T (2008). De-phosphorylation of GR at Ser203 in nuclei associates with GR nuclear translocation and GLUT5 gene expression in Caco-2 cells. Arch Biochem Biophys 475: $1-6$.

Tanti A, Rainer Q, Minier F, Surget A, Belzung C (2012). Differential environmental regulation of neurogenesis along the septo-temporal axis of the hippocampus. Neuropharmaco$\log y$ 63: 374-384.

Tatro ET, Everall IP, Masliah E, Hult BJ, Lucero G, Chana G et al (2009). Differential expression of immunophilins FKBP51 and 


\section{Stress Induces Alterations on GR and FKBP5}

$G$ Guidotti et al

FKBP52 in the frontal cortex of HIV-infected patients with major depressive disorder. J Neuroimmune Pharmacol 4: 218-226.

Trajkovska V, Kirkegaard L, Krey G, Marcussen AB, Thomsen MS, Chourbaji S et al (2009). Activation of glucocorticoid receptors increases 5-HT2A receptor levels. Exp Neurol 218: 83-91.
Wang Z, Frederick J, Garabedian MJ (2002). Deciphering the phosphorylation "code" of the glucocorticoid receptor in vivo. J Biol Chem 277: 26573-26580.

Warner-Schmidt JL, Flajolet M, Maller A, Chen EY, Qi H, Svenningsson P et al (2009). Role of p11 in cellular and behavioral effects of 5-HT4 receptor stimulation. J Neurosci 29: 1937-1946. 